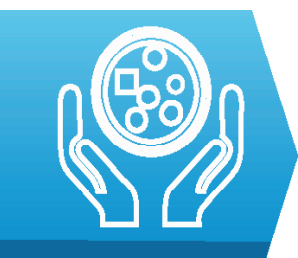

ONCOLOGY

\title{
Updates on the 2016 World Health Organization Classification of Pediatric Tumors of the Central Nervous System - a systematic review
}

\author{
Maria Margareta Cosnarovici ${ }^{1}$, Rodica Voichița Cosnarovici ${ }^{2}$, \\ Doina Piciu ${ }^{1,2}$
}

1) Iuliu Haţieganu University of Medicine and Pharmacy, Cluj-Napoca, Romania

2) "Prof. Dr. Ion Chiricuță" Institute of Oncology, Cluj-Napoca, Romania
DOI: $10.15386 / \mathrm{mpr}-1811$

Manuscript received: 10.07 .2020

Received in revised form: 31.10 .2020

Accepted: 17.03.2021

Address for correspondence:

rodicacosnarovici@yahoo.com

This work is licensed under a Creative Commons Attribution-NonCommercialNoDerivatives 4.0 International License

\begin{abstract}
Tumors of the central nervous system (CNS) represent the main cause of death through solid tumors in children and the second most frequent neoplasm in this patient group. The poor survival rate is due to many factors, such as the large diversity of morphological features, the particular micro-environmental characteristics of the nervous tissue, the relative rareness in relation to other childhood diseases, which leads to late diagnosis and the limited effectiveness of the available treatment options. Up until 2016, brain tumors were classified according to their histologic features. The new 2016 World Health Organization (WHO) Classification of CNS tumors incorporates molecular features, alongside the immunohistology, in order to provide a more accurate understanding of the disease. The treatment consists of surgery, radiation therapy and chemotherapy. We decided to review the literature on this pathology, in order to show the importance of the recent discoveries in this field.
\end{abstract}

Keywords: brain tumors, children, molecular diagnosis, prognosis, treatment

\section{Introduction}

Pediatric tumors of the CNS represent the main cause of death in children aged 0-15 years, and the second most frequent neoplasm in this patient group, being surpassed only by leukemia. They represent a strenuous challenge for the pediatric oncologist, regarding the diagnosis and treatment, but also the management of the side effects or sequelae [1].

The causes of brain tumors in children can be divided into two broad groups: hereditary and non-hereditary causes. Hereditary causes and the exposure to ionizing radiation constitute the known causes for pediatric tumors of the CNS. Certain syndromes are known to be associated with the development of brain tumors. Type 1 neurofibromatosis is the most common, but other syndromes may also be the cause, such as: neurofibromatosis type 2 , tuberous sclerosis type 1 and 2, von Hippel Lindau syndrome, Li Fraumeni syndrome, nevoid basal cell carcinoma syndrome, Turcot's syndrome, ataxia-telangiectasia syndrome and Down syndrome [2,3]. The non-genetic factors are represented by: therapeutic exposure to ionizing radiation, in utero- exposure to an atomic explosion, immunologic deficiency states (HIV, post transplantation immunodeficiency), tobacco smoke, exposure to organic compounds or nitrosamines [4]. From an anatomical point of view, brain tumors can be placed in two categories: supratentorial and infratentorial lesions. The most frequent supratentorial tumors are astrocytomas, ependymomas, gliomas, embryonal tumors, choroid plexus tumors, meningiomas, and germ cell tumors. The most frequent infratentorial tumors are: medulloblastomas, astrocytomas, ependymomas and gliomas [5].

From 2007 until 2016, brain tumors 
were classified according to their immunohistochemical features. The new 2016 World Health Organization (WHO) Classification of Tumors of the Central Nervous System adds molecular parameters to histology, thus providing a more accurate diagnosis and prognosis [6]. Several entities have been defined by the new classification, while others have been removed. An important addition has been made regarding glioblastomas. The new classification divides glioblastomas according to the isocitrate dehydrogenase $1 / 2$ (IDH) gene status, into wild type and mutant glioblastoma. In adults, IDH mutant glioblastoma patients have a 5 -year-old survival rate of approximately $80 \%$. However, in children the role of the IDH gene status has yet to be proven. Another newly defined entity is the diffuse midline glioma-histone H3 K27M mutant. The K27M mutation is a missense mutation, which leads to the alteration of histone 3, resulting in two different variants: H3.3 and H3.1. These mutations lead to epigenetic alterations of the histones and block glial cells from differentiating, thus becoming oncogenic promoters. Children suffering from diffuse midline glioma-H3 K27M mutant, have a worse prognosis than the ones without the mutation [7]. The nosological shift in defining ependymomas has led to the addition of ependymoma-RELA fusion positive, to the classification. This entity accounts for almost $70 \%$ of all supratentorial childhood ependymomas and has a poor prognosis. Following the development of the RELA fusion, the NFkB pathway is pathologically activated, resulting in methylation deficiencies. RELA fusion positive ependymomas have a worse prognosis than the others. The classification of medulloblastomas has also suffered some changes, consisting of the addition of two new genetically different tumors: WNT-activated and Sonic Hedgehog (SHH)-activated medulloblastoma. The WNT-activated and SHH-activated medulloblastoma are mutually exclusive and have different disease outcomes. WNT- medulloblastomas are usually diagnosed in children older than 5 years and have a favorable prognosis, while $\mathrm{SHH}$-activated tumors develop in children younger than 4 years old, and are associated with poor survival rates [8]. The embryonal tumor with multilayered rosettes, C19MC-altered, is also one of the entities newly added to the 2016 classification. This comprises three histologically diverse, but molecularly similar tumors: embryonal tumor with abundant neuropil and true rosettes (ETANTR), ependymoblastoma (EBL) and primitive neuroectodermal tumor (PNET). The similarity consists of: poorly differentiated cellular populations, of the presence of multilayered rosettes and the amplification of C18MC locus [9].

The sole purpose of this classification at the present time is to facilitate an accurate diagnosis and prognosis. In the future, the aim of identifying the molecular patterns of these tumors, is to provide targeted therapies, in order to improve survival rates for this highly aggressive disease [10].
The aim of this paper is to review the literature on this pathology, in order to show the importance of the recent discoveries in this field and to highlight the importance of the molecular diagnosis.

\section{Methods}

The research was performed on the PubMed database. The following keywords were used for the initial search: "malignant brain tumors, children". Only articles published between 2016 and 2020 were taken into account. We retrieved 873 papers, both reviews and clinical trials. We added the following filters: "Full text", "English", "Child:0-18 years", "Cancer", thus obtaining 556 results. Two exclusion criteria were applied: children only receiving palliative care, side effects (this will be the topic of a future study). As a result, we obtained 442 papers.

\section{Results \\ Medulloblastoma}

Medulloblastoma is the most frequent malignant pediatric brain tumor, representing approximately $20 \%$ of all CNS cancers in children aged 0-14 years. Medulloblastomas have a higher occurrence between the ages 3-4 and between 8-10 years of age and boys develop the tumor 3 times more often the girls [11]. The origin of this tumor is thought to be in the cerebellar stem cells, that have not yet progressed towards differentiation. The metastases spread in the CNS through the cerebrospinal fluid, but can also arise in extra neural tissues. There is a well-documented association between medulloblastoma and certain syndromes: Gorlin syndrome (PCTH1 gene and SUFU gene mutations), Turcot syndrome (APC gene mutation), Li-Fraumeni syndrome (TP53 gene mutation).

The new WHO 2016 Classification of brain tumors divides medulloblastomas into 4 types, according to the immunohistological but also molecular characteristics: WNT/ $\beta$-catenin, Sonic Hedgehog, Group 3, and Group 4. Within these 4 groups certain transcriptionally distinct subgroups can be identified. Recurrent signaling pathways disrupted in medulloblastomas are: TGF- $\beta$, MYC, Notch and BMP. Mutations present in certain individuals with medulloblastoma are: SMARCA4, DDX3X, PTCH1, MLL2, CTDNEP1, KDM6A, TBR1 [12]. WNT activated medulloblastomas most frequently arise in children aged 6-10 years and have good overall survival rates (approximately 90\%). It is thought that WNT activated medulloblastomas have good survival rates due to the defective blood brain barrier in these tumors, which allows chemotherapy to reach the lesion more efficiently [13]. SHH activated medulloblastomas arise in infants and adults and have poorer survival rates compared to the WNT group (approximately 70\%). Within this category, patients are divided according to the TP53 gene status into high and standard risk groups. Patients exhibiting the TP53 
gene mutation have a higher risk than those with wild type TP53 gene status. Groups 3 and 4 are both WNT and SHH negative. They express a wide range of genetic alterations, and are still under study. Group 4 medulloblastomas have survival rates of approximately $90 \%$, while group 3 tumors have a much lower survival rate of approximately $50 \%$ [14].

The symptoms at presentation may include: headache, nausea, photophobia, ataxia, dysmetria, wide based and staggering gait, diplopia, strabismus. The golden standard for diagnosis is brain and spinal cord MRI and cerebrospinal fluid analysis. Treatment options include surgery, radiation therapy and chemotherapy. The aim of the surgical treatment is to obtain full resection of the tumoral mass and to reestablish the flow of the cerebrospinal fluid. Depending on the surgical result, age and risk group, patients follow different radiation therapy regimens. If the residual tumor volume is smaller than $1.5 \mathrm{~cm}^{2}$, and there are no metastases, the risk is average. If the patient is metastatic at diagnosis, or if the residual volume is greater than 1.5 $\mathrm{cm}^{2}$, the risk is high. Patients under 3 years of age do not undergo radiation therapy. Older patients receive external radiation to the craniospinal axis (ranging from 23.4 to 36 Gy) and a boost to the posterior fossa (54-55,8 Gy) [15]. The used chemotherapy regimen is HIT2000, which allows for a differentiated treatment according to different risk categories, and includes: Vincristine, Cyclophosphamide, Methotrexate, CCNU, Etoposide, Carboplatin. Second line chemotherapy is represented by the following protocols: HIT REZ, TEMIRI. The MEMMAT trial has prooven the efficiency in treating metastasis or local recurrent disease with metronomic therapy. High dose chemotherapy continued with autologous bone marrow transplantation is a valid option for treating the relapse or infants. The treatment related side effects include: posterior fossa syndrome, hearing deficiency, endocrine deficiency, cerebrovascular disease or a second malignancy. After the end of the oncological treatment, patients need to be monitored through brain and spinal cord MRI every 3 months for the first two years, every 4 months in the $3^{\text {rd }}$ and $4^{\text {th }}$ year and then every 6 months [16].

Novel treatment options currently under study include: 177-Lu-DTPA-Omburtamab (ClinicalTrials. gov identifier NCT04167618), Vismodegib/GDC-0449 (ClinicalTrials.gov identifier NCT01239316), Silmitasertib Sodium/CX-4945 (ClinicalTrials.gov identifier NCT03904862).

\section{Embryonal tumors}

The embryonal tumors of the central nervous system are a large group of histologically diverse neoplasms. They are poorly differentiated and highly aggressive, accounting for approximately $15 \%$ of all pediatric brain tumors. The new WHO 2016 classification of brain tumors uses molecular parameters in addition to histology and groups the following entities as embryonal brain tumors: medulloblastoma, atypical rhabdoid/ theratoid tumors(AT/RT), pinealoblastoma, embryonal tumor with multiple rosettes (ETMR)/C19MC-altered tumors, medulloepitheliomas, CNS neuroblastoma, CNS ganglioneuroblastoma, CNS embryonal tumor non otherwise specified (NOS), CNS embryonal tumor with rhabdoid features.

\section{a. Embryonal tumors with multiple rosettes (ETMR)/C19MC-altered}

The embryonal tumor with multiple rosettes (ETMR), C19MC-altered is a new entity acknowledged by the 2016 WHO classification of CNS tumors. It comprises 3 histologically different malignancies formerly known as embryonal tumor with abundant neuropil and true rosettes (ETANTR), ependymoblastoma and primitive neuroectodermal tumor. Although histologically heterogenous, these tumors have a similar molecular landscape, defined by the amplification of the $\mathrm{C} 19 \mathrm{MC}$ region on chromosome 19 (19q13.42) [9]. ETMR-s are highly aggressive malignancies (WHO grade IV), that primarily affect children younger than 4 years of age, and despite the complex treatment options, have a grim prognosis (5-year event free survival rates are 19-38\%) [17].

The clinical presentation may consist of: nausea, vomiting, seizures, cranial nerve palsy, weakness, ataxia, motor or sensory impairment [18]. The golden standard for diagnosing and staging AT/RT is contrast enhanced brain and spinal cord MRI. The treatment consists of: surgery, chemotherapy, radiation therapy and high dose chemotherapy followed by stem cell rescue. The surgical treatment aims to fully resect the tumor and to reestablish the flow of the cerebrospinal fluid. For children older than 3 years of age, the golden standard is represented by intensity modulated radiation therapy (IMRT). The standard dose for craniospinal axis radiation is usually $36 \mathrm{~Gy}$, while the dose used in tumor bed radiation is $56 \mathrm{~Gy}$. In children younger than 3 years of age, radiation to the craniospinal axis could lead to life long side effects, therefore other treatment options are under study.

Bailey reports favorable results in three children treated with 131I-Omburtamab infused intraventricularly through Ommaya reservoirs. Targeted radioimmunotherapy represents one promising new treatment option, that requires further studies [19].

\section{b. Atypical rhabdoid/theratoid tumors}

Atypical rhabdoid/theratoid tumors represent a highly agressive type of embryonal tumor, accounting for aproximately $2 \%$ of all childhood brain neoplasms. Roughly $2 / 3$ of the patients are younger that 3 years of age at diagnosis [20]. The pathognomonic genetic alteration is represented by mutations of SWI/SNF related, matrix associated, actin-dependent regulator of chromatin, subfamily B, member 1 gene (SMARCB1). In rare cases, which lack the SMARCB1 gene, SWI/SNF related, matrix associated, actin-dependent regulator of chromatin, subfamily A, member 4 (SMARCA4) mutations can be identified. Both 
SMARCB1 and SMARCA4 lead to alterations of the SWI/SNF pathway, resulting in alterations of the genomic landscape. There are three types of molecularly- defined subgroups of atypical rhabdoid/theratoid tumors: AT/RTTYR, AT/RT-SHH, AT/RT-MYC. Within these groups, the locations in the brain, as well as the gene expression are simmilar. AT/RT-TYR subgroup is characterised by young age at the time of diagnosis (0-1 years), infratentorial location, SMARCB1 deletions in most patients, high expression of Microphthalmia-associated transcription factor (MITF), Tyrosinase gene (TYR) and Dopachrome Tautomerase gene (DCT) and a hypermethylated genome. AT/RT-SHH subgroup exhibits SMARCB1 deletion in a few cases, both supra- and ifra-tentorial locations, Sonic Hedgehog (SHH) signaling expression, hiperexpression of MYCN and GLI2, NOTCH signaling activation and a hypermethylated genome. Tumors belonging to the AT/ RT-MYC subgroup most frequently arrise in older children and are located supratentorially. They are characterised by SMARCB1 deletion in a few of the cases, highly expressed MYC oncogene, HOX cluster and HOX Transcript Antisense RNA (HOTAIR) genes [21]. The molecular simmilarities within these subgroups suggest that AT/RT may derive from different precursor cells.

Symptoms may include: fatigue, nausea, headache, ataxia, wide based and staggering gait, cranial nerve palsy, seizures, behavioral alterations [22]. The golden standard for diagnosing and staging AT/RT is contrast enhanced brain and spinal cord MRI. The multimodality treatment consists of surgery, radiation therapy and chemotherapy. Obtaining full gross resection of the tumor leads to better prognosis. Radiation therapy doses range from 24.0 to 36.0 Gy to the craniospinal axis, and from 30.0 to 56.0 to the tumor bed. In patients younger than 3 years of age, RT doses used on the tumor bed are significantly lower, ranging from 41.8 to $50.4 \mathrm{~Gy}$. The challenges in using craniospinal axis radiation in children younger than 3 years old reside in the severe neurocognitive and endocrine sequalae. Postponing radiation therapy by administering chemotherapy has shown no effectiveness in treating AT/RT [23]. Intrathecal administration of chemotherapy and concomitant radiation of the tumor bed has proven itself effective, but further studies must be conducted. Chemotherapy consists of Ifosfamide, Cisplatin, and Etoposide (ICE) for children younger than 3 years old, and Carboplatin, Thiotepa, and Etoposide, or Cyclophosphamide and Melphalan (HDCT), followed by autologous bone marrow cell rescue. Long term event free survival rates are dismal, the median survival being 9.4 months.

Tyrosine kinase inhibitors show great promise in treating AT/RT. Retinoids and Cyclin-inhibitors may also prove themselves to be efficient therapeutic agents in the future [24].

\section{Ependymoma}

The ependymoma is the third most frequent brain neoplasm in children, accounting for $6-10 \%$ of all child brain tumors. The cell of origin is thought to be the epithelial cells lining the cerebral ventricles or the spinal cord central canal. In children, the ependymoma develops in the brain, in $90 \%$ of the cases, of which two thirds are infratentorial and one third supratentorial tumors. The initial symptoms may include: fatigue, nausea, headache, ataxia, wide based and staggering gait, cranial nerve palsy, seizures and behavioral alterations [25].

The WHO 2016 classification of brain tumors divides ependymomas into 3 grades, according to the aggressiveness in the histological pattern. Grade I includes subependymomas and myxopapillary ependymomas. Grade II includes the classic ependymoma (cellular, papillary and tanycytic subtypes) and the ependymoma, RELA fusion positive. Grade III is represented by anaplastic ependymomas. Molecularly, ependymomas are classified into 4 categories: RELA fusion positive, YAP-1 fusion positive, PF-A and PF-B [26]. More than 70\% of all supratentorial ependymomas in children express the RELA-fusion gene, thus proving that all supratentorial tumors should be tested for the RELA-fusion gene. Almost $3 / 4$ of RELA-fusion positive ependymomas have an anaplastic histology. RELA fusion positive ependymomas have a grim prognosis compared to the YAP1-positive ependymomas. Other prediction factors are: histologic grade, p53 expression, young age and extent of resection.

The golden standard for diagnosing and staging ependymomas is contrast enhanced brain and spinal cord MRI. Treatment options include surgery, radiation therapy and chemotherapy. The aim of the surgical treatment is to obtain full resection of the tumoral mass and to reestablish the flow of the cerebrospinal fluid. If the tumor is nonresectable, a biopsy should be performed. Depending on the surgical result, age and risk group, patients follow different radiation therapy regimens. IMRT is the golden standard for treating children older than 3 years of age, with doses ranging from 50.4 to 59.4 Gy. Treating children under 3 years with IMRT, could lead to severe neurocognitive and sensory side effects. The consensus of postponing radiation therapy in children younger than 3 , has led to poor therapeutic results, particularly in cases where there is more than 1 year between surgery and radiation treatment. Proton-beam radiation therapy has the advantage of sparing healthy tissue, in comparison to photon-beam radiation therapy. Some studies suggest that children treated with proton-beam radiation therapy have comparable 3-year progression free survival rates as children treated with IMRT [27]. The first line chemotherapy regimen is HIT2000. Second line chemotherapy is represented by the HIT REZ protocol. Follow up protocols recommend monitoring patients through brain and spinal cord MRI every 3 months for the first two years, every 4 months in the $3^{\text {rd }}$ and ${ }^{\text {th }}$ year and then every 6 months [8].

Some of the treatment options under investigation 
are: Infusion of 5-Azacytidine (5-AZA) Into the Fourth Ventricle in Patients With Recurrent Posterior Fossa Ependymoma (NCT03572530), Immunotherapy for Recurrent Ependymomas in Children Treatment for Recurrent Ependymomas Using HLA-A2 Restricted Tumor Antigen Peptides in Combination With Imiquimod (NCT01795313), Everolimus for Children With Recurrent or Progressive Ependymoma (NCT02155920).

\section{Low grade gliomas}

Low grade gliomas are the most common pediatric brain tumors, with a peak of occurrence between ages 6 and 11. They originate in the glial cells of the central nervous system and have an indolent behavior, being classified as WHO grade I and II. This heterogeneous group of tumors encompasses, according to the new WHO 2016 classification, a number of 10 histologically diverse neoplasms. These neoplasms are represented by: pilocytic astrocytoma (grade I), subependymal giant cell astrocytoma (grade I), IDH mutant diffuse astrocytoma (grade II), IDH mutant or $1 \mathrm{p} / 19 \mathrm{q}$ deletion oligodendroglioma (grade II), pleomorphic xanthoastrocytoma (grade II), angiocentric glioma (grade I), choroid glioma of the third ventricle( grade I or II), gangliocytoma (grade I), ganglioglioma (grade I or II), desmoplastic infantile astrocytoma and ganglioglioma (grade I) [28].

The most frequent localization of this neoplasm is in the posterior fossa, accounting for $15-25 \%$ of the cases. Tumors arise in the brain hemispheres with a frequency of $10-15 \%$, making optic pathways the rarest localization (6\%). Symptoms may include: nausea, headache, seizures, motor deficit, loss of sight, nystagmus, ataxia, gait. Patients suffering from neurofibromatosis and tuberous sclerosis have a predisposition for developing low grade gliomas. Approximately $20 \%$ of type 1 neurofibromatosis patients develop gliomas at one point in their life, however these tumors are less aggressive than their counterparts in patients not suffering from NF1 [29].

The golden standard for diagnosing and staging low grade gliomas is contrast enhanced MRI of the brain and spinal cord. The staging is done according to the Chang system, into 4 stages. The treatment options include surgery, chemotherapy and radiation therapy. The aim of surgery is to obtain full resection of the tumoral mass. If this is not obtained, patients may undergo nonsurgical treatments. The line 1 chemotherapy regimen used is SIOP-LGG 2004. The line 2 chemotherapy regimens are: SIOP LGG, Vinblastine, Temozolomide, Bevacizumab/IRI. The radiation treatment consists of IMRT. For children over 4 years of age the mean doses are 54 Gy for brain tumors and 50.4 Gy for spinal tumors. There is a lot of controversy around the matter of treating children under 4 years of age with IMRT. Proton beam radiation therapy is under study. Patients should be followed up through MRI every 3 months for the first 2 years, every 6 months for the next 3 years and then once a year for the next 5 years [10].
New targeted tratment options are under study: Dabrafenib, Trametinib and Hydroxychloroquine for Patients With Recurrent LGG or HGG With a BRAF Aberration (NCT04201457), Bevacizumab and Irinotecan in Treating Young Patients With Recurrent, Progressive, or Refractory Glioma, Medulloblastoma, Ependymoma, or Low Grade Glioma (NCT00381797). Polyepitopic vaccines are also under study for this pathology: A Pilot Study of Glioma Associated Antigen Vaccines in Conjunction with Poly-ICLC in Pediatric Gliomas (NCT01130077).

\section{High grade gliomas}

High grade gliomas are highly aggressive tumors and account for 3-7\% of all pediatric brain tumors. The peak incidence is during adolescence. The corresponding WHO grades are III and IV. They are derived from astrocytes and oligodendroglial cells. The group of high-grade gliomas comprises a number of 7 histologically diverse neoplasms. They are represented by: anaplastic astrocytoma (grade III), IDH wild type glioblastoma (grade IV), IDH mutant glioblastoma (grade IV), H3K27M mutant difuse midline glioma (grade IV), IDH mutant and with $1 \mathrm{p} / 19 \mathrm{q}$ codeletion anaplastic oligodendroglioma (grade III) and pleomorphic anaplastic xanthoastrocytoma (grade III) [30]. Genetic testing includes the following genes: IDH, BRAF (epithelioid glioblastoma), MYC (glioblastoma with PNET components), EGFR (small cell and granular glioblastoma), H3K27M (difuse midline glioma) [31].

Symptoms appear over a short period of time, due to the high growth rate of the tumor. Symptoms may include: nausea, headache, seizures, motor deficit, loss of sight, nystagmus, ataxia, gait. The golden standard for diagnosing and staging high grade gliomas is contrast enhanced MRI of the brain and spinal cord. Surgery is the main treatment tool. Obtaining full resection of the tumor is often impossible because of the involvement of vital structures. Chemotherapy consists of HIT -GBM D, temozolomide and Bevacizumab with Irinotecan. Radiation therapy for children older than 3 years of age consists of IMRT. Doses range from 54 to 60 Gy. Patients suffering from high grade gliomas have a poor prognosis. The 5-year-old disease free survival rates range from 15 to $35 \%$. Post treatment follow up consists of brain and spinal cord MRI every 3 months [32].

There are several tyrosine-kinase inhibitors under study, in combination with other antineoplastic agents: Study of the Combination of Crizotinib and Dasatinib in Pediatric Research Participants with Diffuse Pontine Glioma and High-Grade Glioma (NCT01644773), Pilot Study of Cabozantinib for Recurrent or Progressive High-Grade Glioma in Children (NCT02885324). Other novel therapies under investigation are: Intra-tumoral Injection of Natural Killer Cells in High-Grade Gliomas (NCT04254419), Adoptive Cellular Therapy in Pediatric Patients with Highgrade Gliomas (NCT03334305), Antineoplaston Therapy in Treating Children with Recurrent or Refractory HighGrade Glioma (NCT00003535). 


\section{Germ cell tumors of the brain}

Germ cell tumors are a relatively rare disease in children and represent approximately $4 \%$ of all pediatric brain tumors. They are most common between ages 1015 years. They occur due to a faulty migration of the mesoderm and most commonly develop along the midline, either involving or in the vicinity of the pituitary gland and suprasellar region.

The WHO 2016 classification divides this pathology into 2 large categories: pure germ cell tumors and mixed germ cell tumors. There are 7 types of mixed germ cell tumors: embryonal carcinoma, yolk sack tumors, germinoma, teratoma, teratoma with malignant transformation and mixed germ cell tumor. Children suffering from Down syndrome or Klinefelter syndrome have a predisposition for developing germ cell tumors. Signs and symptoms include: headache, nausea, vomiting, endocrinologic deficiencies, loss of sight, bitemporal hemianopsia, diplopia [33].

The golden standard in staging and diagnosing germ cell tumors is represented by contrast enhanced brain and spinal cord MRI, followed by cerebrospinal fluid Alpha-fetoprotein (AFP) and $\beta$-Human Chorionic Gonadotropin ( $\beta$-HCG) evaluation. The treatment options are represented by surgery, chemotherapy and radiation. The aim of the surgery is to obtain full resection of the tumor and to reestablish the flow of the cerebrospinal fluid. The chemotherapy regimen in use is SIOP CNS GCT II. Radiation therapy protocols are very different and are established according to histology, the surgical result and the age. Total radiation doses range from 24 to 54 Gy. Patients should be followed up through MRI and serum markers every 3 months during the first year, every 6 months for the following 2 years and then once a year for at least 2 more years [34].

Some of the ongoing studies trying to identify new treatment options are: Gemcitabine, Paclitaxel and Oxaliplatin (NCT01270724), Chemotherapy Followed by Radiation Therapy in Treating Younger Patients With Newly Diagnosed Localized Central Nervous System Germ Cell Tumors (NCT01602666).

\section{Conclusion}

In the past decades, the survival rates in pediatric malignancies have been significantly improved. Unfortunately, childhood brain tumors do not fall under the same category. This is due to late diagnosis, rapid tumor extension and the shortcoming of antineoplastic agents capable of penetrating the blood-brain barrier. The WHO 2016 classification adds molecular parameters to the classic histology, thus demonstrating that although histologically similar to the adult version, pediatric brain tumors have very different genetic landscapes. Understanding the alterations in the tumoral signaling pathways is not only the key to developing more accessible diagnostic tools, but also a pivotal element in providing personalized targeted therapies. The current trend is to replace genetic examinations with the cheaper, more feasible alternative represented by immunohistochemistry. This may however prove to be a difficult task, since providing a correct genomic and epigenetic description of these tumors still requires delicate and complex techniques. The newly emerged diagnostic subgroups do not have any implications in the therapeutic approach yet as they only provide a more accurate diagnosis and prognosis. Although somewhat difficult to understand and identify, these new subgroups will lead to a more personal treatment approach, consisting of surgery, radiation therapy, chemotherapy and small molecule inhibitors. As the targeted molecular therapies are slowly taking over the classical oncological therapies, childhood brain tumors will most likely benefit from this more efficient treatment option in the near future.

\section{References}

1. Jaimes C, Poussaint TY. Primary Neoplasms of the Pediatric Brain. Radiol Clin North Am. 2019;57:1163-1175.

2. Evans DGR, Salvador H, Chang VY, Erez A, Voss SD, Schneider KW, et al. Cancer and central nervous system tumor surveillance in pediatric neurofibromatosis 1. Clin Cancer Res. 2017;23:e46-e53.

3. Michaeli O, Tabori U. Pediatric high grade gliomas in the context of cancer predisposition syndromes. J Korean Neurosurg Soc. 2018;61:319-332.

4. Ostrom QT, Adel Fahmideh M, Cote DJ, Muskens IS, Schraw JM, Scheurer ME, et al. Risk factors for childhood and adult primary brain tumors. Neuro Oncol. 2019;21:1357-1375.

5. AlRayahi J, Zapotocky M, Ramaswamy V, Hanagandi P, Branson H, Mubarak W, et al. Pediatric brain tumor genetics: What radiologists need to know. Radiographics. 2018;38:2102-2122.

6. Louis DN, Perry A, Reifenberger G, von Deimling A, Figarella-Branger D, Cavenee WK, et al. The 2016 World Health Organization Classification of Tumors of the Central Nervous System: a summary. Acta Neuropathol. 2016;131:803-820.

7. Garcia MA, Solomon DA, Haas-Kogan DA. Exploiting molecular biology for diagnosis and targeted management of pediatric low-grade gliomas. Future Oncol. 2016;12:14931506.

8. Klonou A, Piperi C, Gargalionis AN, Papavassiliou AG. Molecular Basis of Pediatric Brain Tumors. Neuromolecular Med. 2017;19:256-270.

9. Horwitz M, Dufour C, Leblond P, Bourdeaut F, Faure-Conter C, Bertozzi AI, et al. Embryonal tumors with multilayered rosettes in children: the SFCE experience. Childs Nerv Syst. 2016;32:299-305.

10. Wang SS, Bandopadhayay P, Jenkins MR. Towards Immunotherapy for Pediatric Brain Tumors. Trends Immunol. 2019;40:748-761.

11. Massimino M, Biassoni V, Gandola L, Garrè ML, Gatta G, 
Giangaspero F, et al. Childhood medulloblastoma. Crit Rev Oncol Hematol. 2016;105:35-51.

12. Cambruzzi E. Medulloblastoma, WNT-activated/SHHactivated: clinical impact of molecular analysis and histogenetic evaluation. Childs Nerv Syst. 2018;34:809-815.

13. Cavalli FMG, Remke M, Rampasek L, Peacock J, Shih DJH, Luu B, et al. Intertumoral Heterogeneity within Medulloblastoma Subgroups. Cancer Cell. 2017;31:737754.e6.

14. Archer TC, Mahoney EL, Pomeroy SL. Medulloblastoma: Molecular Classification-Based Personal Therapeutics. Neurotherapeutics. 2017;14:265-273.

15. Padovani L, Horan G, Ajithkumar T. Radiotherapy Advances in Paediatric Medulloblastoma Treatment. Clin Oncol (R Coll Radiol). 2019;31:171-181.

16. Guerrini-Rousseau L, Dufour C, Varlet P, Masliah-Planchon J, Bourdeaut F, Guillaud-Bataille $\mathrm{M}$, et al. Germline SUFU mutation carriers and medulloblastoma: clinical characteristics, cancer risk, and prognosis. Neuro Oncol. 2018;20:1122-1132.

17. Choi SH, Kim SH, Shim KW, Han JW, Choi J, Kim DS, et al. Treatment outcome and prognostic molecular markers of supratentorial primitive neuroectodermal tumors. PLoS One. 2016;11:e0153443.

18. Wang B, Gogia B, Fuller GN, Ketonen LM. Embryonal Tumor with Multilayered Rosettes, C19MC-Altered: Clinical, Pathological, and Neuroimaging Findings. J Neuroimaging. 2018;28:483-489.

19. Bailey K, Pandit-Taskar N, Humm JL, Zanzonico P, Gilheeney $\mathrm{S}$, Cheung NK V, et al. Targeted radioimmunotherapy for embryonal tumor with multilayered rosettes. J Neurooncol. 2019;143:101-106.

20. Al-Hussaini M, Dissi N, Souki C, Amayiri N. Atypical teratoid/ rhabdoid tumor, an immunohistochemical study of potential diagnostic and prognostic markers. Neuropathology. 2016;36:17-26.

21. Babgi M, Samkari A, Al-Mehdar A, Abdullah S. Atypical Teratoid/Rhabdoid Tumor of the Spinal Cord in a Child: Case Report and Comprehensive Review of the Literature. Pediatr Neurosurg. 2018;53:254-262.

22. Biswas A, Kashyap L, Kakkar A, Sarkar C, Julka PK. Atypical teratoid/rhabdoid tumors: challenges and search for solutions. Cancer Manag Res. 2016;8:115-125.

23. Yang WC, Yen HJ, Liang ML, Chen HH, Lee YY, Wong TT, et al. Role of early and aggressive post-operative radiation therapy in improving outcome for pediatric central nervous system atypical teratoid/rhabdoid tumor. Childs Nerv Syst. 2019;35:1013-1020.

24. Frühwald MC, Biegel JA, Bourdeaut F, Roberts CW, Chi $\mathrm{SN}$. Atypical teratoid/rhabdoid tumors-current concepts, advances in biology, and potential future therapies. Neuro Oncol. 2016;18:764-778.

25. Wang L, Liu L, Li H, Wang PP, Hu Z, Wei Y, et al. RELA Fusion in Supratentorial Extraventricular Ependymomas: A Morphologic, Immunohistochemical, and Molecular Study of 43 Cases. Am J Surg Pathol. 2019;43:1674-1681.

26. Onishi S, Yamasaki F, Nakano Y, Takayasu T, Amatya VJ, Kolakshyapati $\mathrm{M}$, et al. RELA fusion-positive anaplastic ependymoma: molecular characterization and advanced MR imaging. Brain Tumor Pathol. 2018;35:41-45.

27. Indelicato DJ, Bradley JA, Rotondo RL, Nanda RH, Logie $\mathrm{N}$, Sandler ES, et al. Outcomes following proton therapy for pediatric ependymoma. Acta Oncol. 2018;57:644-648.

28. Rudà R, Reifenberger G, Frappaz D, Pfister SM, Laprie A, Santarius T, et al. EANO guidelines for the diagnosis and treatment of ependymal tumors. Neuro Oncol. 2018;20:445456.

29. Jones DTW, Kieran MW, Bouffet E, Alexandrescu S, Bandopadhayay P, Bornhorst M, et al. Pediatric low-grade gliomas: next biologically driven steps. Neuro Oncol. 2018;20:160-173.

30. Sturm D, Pfister SM, Jones DTW. Pediatric gliomas: current concepts on diagnosis, biology, and clinical management. J Clin Oncol. 2017;35:2370-2377.

31. Jones C, Karajannis MA, Jones DTW, Kieran MW, Monje M, Baker SJ, et al. Pediatric high-grade glioma: biologically and clinically in need of new thinking. Neuro Oncol. 2017;19:153-161.

32. Braunstein S, Raleigh D, Bindra R, Mueller S, Haas-Kogan D. Pediatric high-grade glioma: current molecular landscape and therapeutic approaches. J Neurooncol. 2017;134:541-549.

33. Bowzyk Al-Naeeb A, Murray M, Horan G, Harris F, Kortmann RD, Nicholson J, et al. Current Management of Intracranial Germ Cell Tumours. Clin Oncol (R Coll Radiol). 2018;30:204-214.

34. Takada A, Ii N, Hirayama M, Toyoda H, Matsubara T, Toyomasu Y, et al. Long-term follow-up of intensive chemotherapy followed by reduced-dose and reduced-field irradiation for intracranial germ cell tumor. J Neurosurg Pediatr. 2019;23:317-324. 